\title{
Multimodal Acquisition of Properties and Structure with Transmission Electron Reciprocal-space (MAPSTER) Microscopy
}

Jim Ciston ${ }^{1}$, Colin Ophus ${ }^{1}$, Peter Ercius ${ }^{1}$, Hao Yang ${ }^{1}$, Roberto dos Reis ${ }^{1}$, Christopher T. Nelson ${ }^{2}$, ShangLin $\mathrm{Hsu}^{2}$, Christoph Gammer ${ }^{3}$, Burak V. Özdöl, ${ }^{4}$ Yu Deng ${ }^{5}$ and Andrew Minor ${ }^{1,2}$

1. National Center for Electron Microscopy, Molecular Foundry, Lawrence Berkeley National Laboratory, Berkeley, USA

2. Department of Materials Science and Engineering, University of California, Berkeley, USA

3. Physics of Nanostructured Materials, Faculty of Physics, University of Vienna, Austria

4. Western Digital Media, Fremont, USA

${ }^{5 .}$ Laboratory of Solid State Microstructure, Nanjing University, Nanjing, P.R. China

There is an ongoing revolution in electron scattering spurred by development of new detector technology; data can be obtained at 1000 times greater speed, enabling innovative new modes of electron microscopy formerly unachievable (Figure 1). In this presentation, I will describe the development of a new experimental capability to simultaneously generate 2D maps of the strain [1], polarization, local distortion and electric fields [2] of materials at unit cell resolution $(<1 \mathrm{~nm})$ or better in a single dataset through Multimodal Acquisition of Properties and Structure in Transmission Electron Reciprocal-space (MAPSTER) Microscopy. These maps can additionally be supplemented by ptychographic reconstructions [3] of the full aberration-corrected electron phase processed form the identical source data.

To demonstrate the feasibility of extracting multiple signals from one MAPSTER dataset, we have experimentally investigated a $\mathrm{SrTiO}_{3} / \mathrm{PbTiO}_{3}$ multilayer heterostructure that has been recently shown to exhibit ferroelectric polarization vortices with potential spintronic applications [4]. With MAPSTER Microscopy, it is possible to simultaneously extract the strain and a polarization-related signal from a single diffraction scan at unit cell resolution with $0.04 \mathrm{~nm}$ pixel sampling (4 mrad convergence semiangle) over $256 \times 256$ probe positions (Figure 2). A set of virtual detectors were defined by precisely fitting the locations of all overlapping discs in the CBED pattern and independently integrating desired regions to generate signals related to conventional bright field and annular bright field signals. By measuring the differential contrast from the broken symmetry of opposing disc overlap regions, a signal related to the local polarization of the material can also be extracted. While this method is not as quantitative as full position averaged CBED (PACBED) fitting, such a measurement demonstrates the limitations of conventional physical detector geometries. We were also able to directly measure the local strain at unit cell resolution by fitting the edges of the overlapping discs [5].

References:

[1] V.B. Ozdol, et al, Applied Physics Letters, 106, 253107 (2015)

[2] Knut Müller, et al, Nature Communications, 5, 5653 (2014)

[3] P. D. Nellist, B. C. McCallum, and J. M. Rodenburg, Nature, 374, 630 (1995)

[4] A. K. Yadav, et al, Nature 530, 198-201 (2016)

[5] Work at the Molecular Foundry, Lawrence Berkeley National Laboratory, was supported by the U.S. Dept. of Energy under Contract \# DE-AC02-05CH11231. CG acknowledges the Austrian Science Fund (FWF):[J3397] and RR acknowledges the support of CAPES/BR Foundation Process No. 1204713-9. 


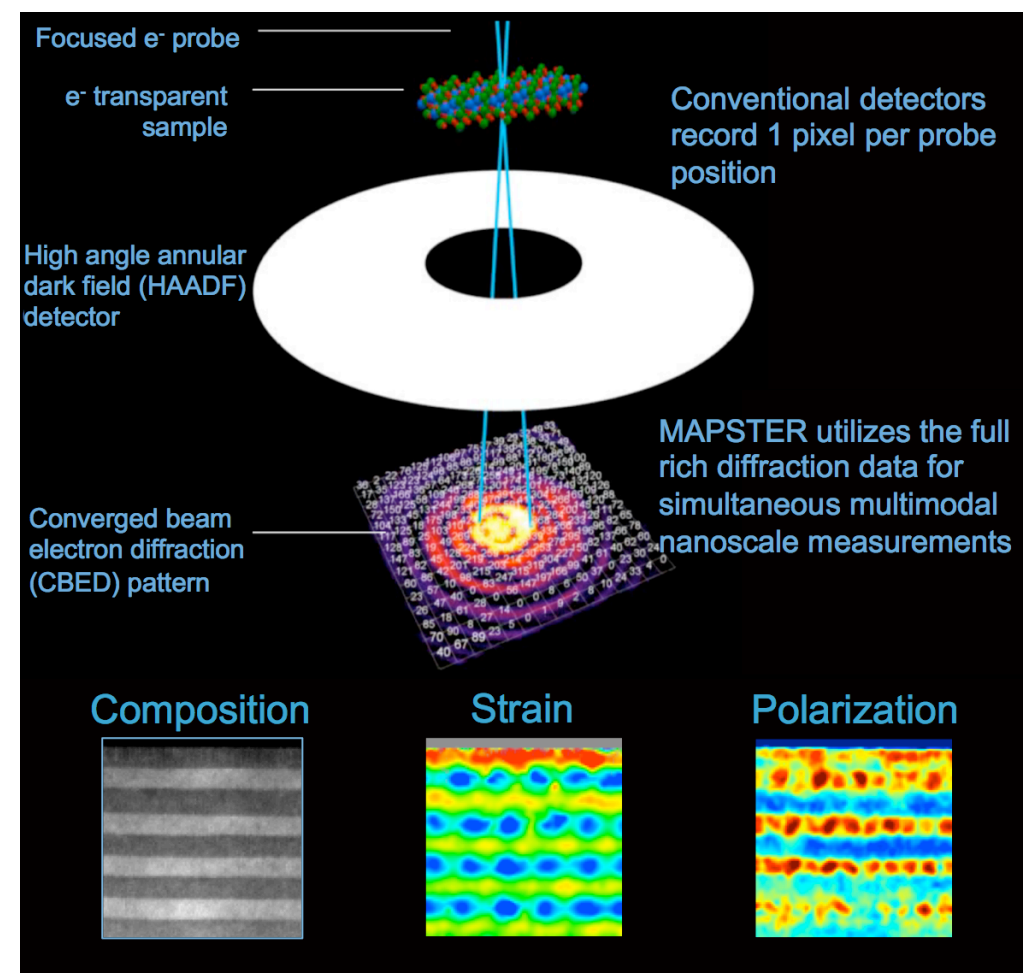

Figure 1. Schematic of MAPSTER Microscopy experimental geometry
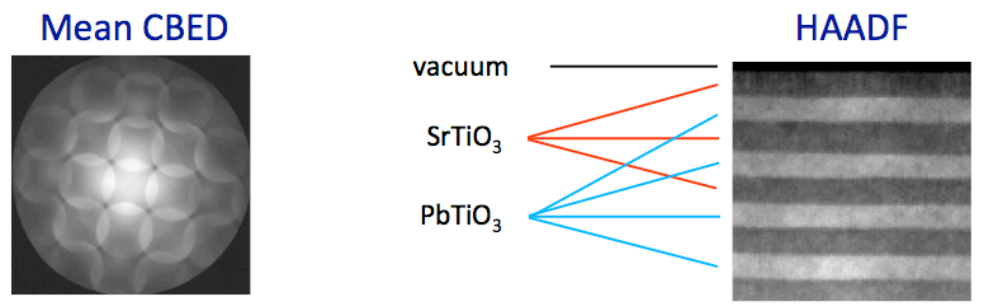

Virtual Detectors

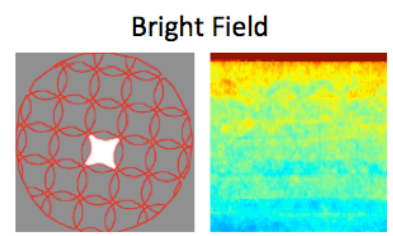

Polarization (x)
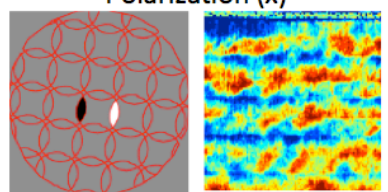

Annular Bright Field
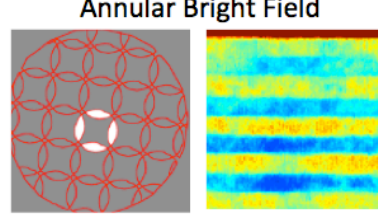

Polarization (y)

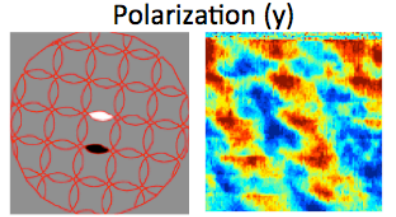

Strain

$\varepsilon_{\mathrm{xx}}( \pm 1 \%)$

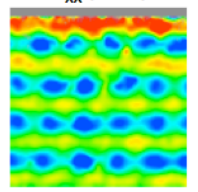

$\varepsilon_{\mathrm{yy}}( \pm 1 \%)$

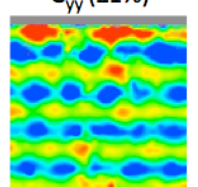

Figure 2. Demonstration of multimodal information acquired from a single MAPSTER dataset (top) Mean PACBED and HAADF image acquired from MAPSTER Microscopy data for a $\mathrm{SrTiO}_{3} / \mathrm{PbTiO}_{3}$ multilayer heterojunction (bottom, left) Images computed from ideal virtual detectors fitted precisely to the disc overlap regions of the recorded CBED data. The differential detectors are sensitive to contributions from polarization, but are not yet fully quantitative (bottom, right). 